\title{
Experiencia de la aplicación de la metodología Flipped-Teaching en la asignatura Concurrencia y Sistemas Distribuidos
}

Estefanía Argente ${ }^{\mathrm{a}}$, Agustín Espinosa ${ }^{\mathrm{a}}$ y Ana García-Fornes ${ }^{\mathrm{a}}$

aUniversitat Politècnica de Valencia (\{eargente,aespinos,agarcia\}@dsic.upv.es)

\begin{abstract}
The Flipped-Teaching methodology inverts the traditional model of teaching, so now students, by themselves, take initial contact with each unit through a series of videos and readings, while classroom sessions mainly focus on resolving exercises, solving doubts, and discussing those aspects that require clarification. In this work, we present the scheduling and methods used in the course "Concurrency and Distributed Systems", a 2nd year mandatory subject of Computer Engineering Degree, following the Flipped-Teaching methodology. This helps us to increase student motivation and involvement as well as improve their self-learning. To this end, we generated a wide visual material of the theoretical part of the course, complemented by guidelines that specify the tasks to perform after viewing each video. The student motivation for this type of approach has been enormous, clearly reflected in their active participation in class and on the good results of the tests conducted so far.
\end{abstract}

Keywords: flipped- teaching, motivation, active learning.

\footnotetext{
Resumen

La metodología de clase inversa (Flipped-Teaching) consiste en invertir el modelo tradicional de docencia, siendo ahora los alumnos quienes, en su casa, tomen contacto inicial con cada unidad temática, a través de una serie de vídeos y lecturas confeccionados por los profesores, mientras que las sesiones de aula se centran en realizar ejercicios, resolver dudas y discutir aquellos aspectos que se requiera clarificar. En este trabajo se presenta la organización de la docencia y los métodos utilizados en la asignatura "Concurrencia y Sistemas Distribuidos", obligatoria de $2^{\circ}$ curso del Grado de Ingeniería Informática, aplicando la metodología Flipped-Teaching, con
} 
Experiencia de la aplicación de la metodología Flipped-Teaching en la asignatura Concurrencia y Sistemas Distribuidos

el propósito de aumentar la motivación e implicación del alumno, así como mejorar su aprendizaje autónomo y significativo. Para ello, se ha generado un amplio material visual de la parte teórica de la asignatura, complementándose con guías que especifican las tareas a realizar tras visualizar cada vídeo. La motivación de los alumnos por este tipo de metodología ha sido enorme, reflejándose claramente en su participación activa durante las clases, así como en los buenos resultados de los exámenes realizados hasta el momento.

Palabras clave: flipped teaching, docencia inversa, motivación, aprendizaje activo.

\section{Introducción}

La metodología de clase inversa (Olaizola, 2014) (Sánchez Rodríguez, 2014), también denominada Flipped-Teaching o Flipped classroom (Jinlei, 2012), consiste en invertir el modelo tradicional de docencia, de modo que la lección magistral habitual del aula se sustituye por un conjunto de materiales en línea, que pueden ser vídeos, lecturas, etc., que el alumno puede consultar, visualizar, etc., mientras que las sesiones de aula se transforman en sesiones fundamentalmente prácticas, con actividades individuales o en grupo, donde el profesor interviene como guía (Ruiz Palmero, 2014). De esta manera, la clase inversa es un método pedagógico en el que se cambian los roles del profesor y alumno dentro y fuera del aula: la clase magistral se lleva a casa, donde el estudiante aprende los conocimientos a través de vídeos y de material multimedia, y el tiempo de clase se utiliza para hacer prácticas, trabajo en equipo y otras dinámicas más colaborativas.

Esta metodología es factible gracias al amplio uso de las nuevas tecnologías. De hecho, gracias a ellas, en los últimos años han aparecido nuevas modalidades de formación (Sánchez Rodríguez, 2014), como el aprendizaje electrónico (electronic learning), el semipresencial (blended learning), el de educación masiva, como los denominados MOOC (Massive Open Online Courses), etc. La clase inversa es una forma de aprendizaje semipresencial (blended learning), donde la asistencia a clases es fundamental, pues en clase se realizan tareas que requieren de mayor interacción y participación con los compañeros o del asesoramiento más personalizado por parte del profesor (Sánchez Rodríguez, 2014).

Durante el curso 2014-2015, la Universitat Politècnica de València ha lanzado una experiencia piloto de clase inversa en un grupo piloto del Grado en Administración y Dirección de Empresas y en otro grupo piloto del Grado en Ingeniería Informática. En el caso del Grado de Ingeniería Informática, impartido en la Escuela Técnica Superior de

(c)) EY-NC-ND 2015, Universitat Politècnica de València 
Ingeniería Informática (ETSINF), este grupo piloto se implantó en todas las asignaturas obligatorias de segundo curso. La asignatura que nos ocupa, Concurrencia y Sistemas Distribuidos (CSD), es una de ellas. Dicha asignatura consta de 6 créditos ECTS, se imparte en el segundo semestre y cubre los siguientes aspectos: principios y técnicas de programación concurrente y de tiempo real; fundamentos de los sistemas distribuidos; y fundamentos de administración de sistemas.

En el curso 2014/15 se ha impartido en 7 grupos de teoría, con 2 sesiones semanales de una hora y media cada una (en total, 15 semanas, es decir, unas 30 sesiones); así como en 14 grupos de laboratorio, de 10 sesiones en total, cada una de hora y media. Además, se ha impartido un grupo extra con la metodología Flipped-Teaching, con 16 alumnos matriculados. Estos alumnos tienen la particularidad de que son alumnos voluntarios, es decir, que se matricularon de forma totalmente voluntaria en esta nueva experiencia, en todas sus asignaturas de segundo curso. En total, en la asignatura hay 379 alumnos matriculados.

En este artículo se presenta la organización de la docencia y los métodos utilizados en esta asignatura, a fin de aumentar la motivación e implicación del alumno, así como mejorar su aprendizaje autónomo y significativo, en el contexto de la metodología Flipped-Teaching.

\section{Objetivos}

La experiencia tiene como objetivo fomentar en los alumnos el aprendizaje activo dentro y fuera del aula, de forma que puedan llegar a las clases con el material trabajado y con mayor disposición a resolver las actividades que se planteen.

También se pretende que tanto alumnos como profesores establezcan una adecuada planificación de las actividades, evitando en lo posible los picos de trabajo. Para ello, los alumnos deberán organizar y planificar su dedicación a la asignatura y los profesores deberán seleccionar los contenidos, desarrollar los recursos y realizar una organización, programación, y planificación temporal óptimas.

\section{Desarrollo de la innovación}

En el modelo de la clase invertida, antes de la clase, el docente produce o selecciona un material digital (video, presentación audiovisual, página web, artículos on-line, etc.), en donde se exponen determinados contenidos del curso y se desarrollan distintos tipos de actividades para verificar la comprensión de los temas (Olaizola, 2014). En la clase, el docente puede aclarar los conceptos más complejos, asistir de forma individual a los alumnos y fomentar el compromiso de los estudiantes con su propio aprendizaje.

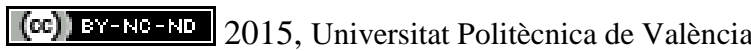


Experiencia de la aplicación de la metodología Flipped-Teaching en la asignatura Concurrencia y Sistemas Distribuidos

En la preparación del material de clase inversa, así como para la impartición de las sesiones correspondientes, hemos colaborado 3 profesores de la asignatura. Así, cada uno se ha encargado de un conjunto de unidades didácticas, para las que realizaba las siguientes tareas:

- Elaboración de vídeos. Por cada unidad didáctica se ha elaborado, en general, una media de unos 4 vídeos, de unos 8 - 10 minutos cada uno. En algunas unidades incluso se ha llegado a generar hasta 10 vídeos, de unos 5-8 minutos cada uno, para explicar de forma precisa cada uno de los conceptos y algoritmos concretos que se trataban en dicha unidad. El formato de los vídeos generados por parte de los tres profesores has sido similar, utilizando principalmente Screencasts generados en Camtasia, aunque también uno de ellos se decantó por realizar vídeos similares a Polimedia (es decir, que incluían la imagen del profesor), pero elaborados en su propio despacho utilizando la herramienta Camtasia.

Todos los vídeos elaborados se han subido a Politube (herramienta de distribución de material audiovisual de la Universitat Politècnica de Valencia) y están disponibles para toda la comunidad universitaria.

A modo de ejemplo, mostramos a continuación una captura de pantalla de uno de los vídeos realizados. Todos estos vídeos son totalmente auto-contenidos, sin referencia explícita a la asignatura, para así poder cumplir con la normativa de “Docencia en Red” de la Universitat Politècnica de Valencia.

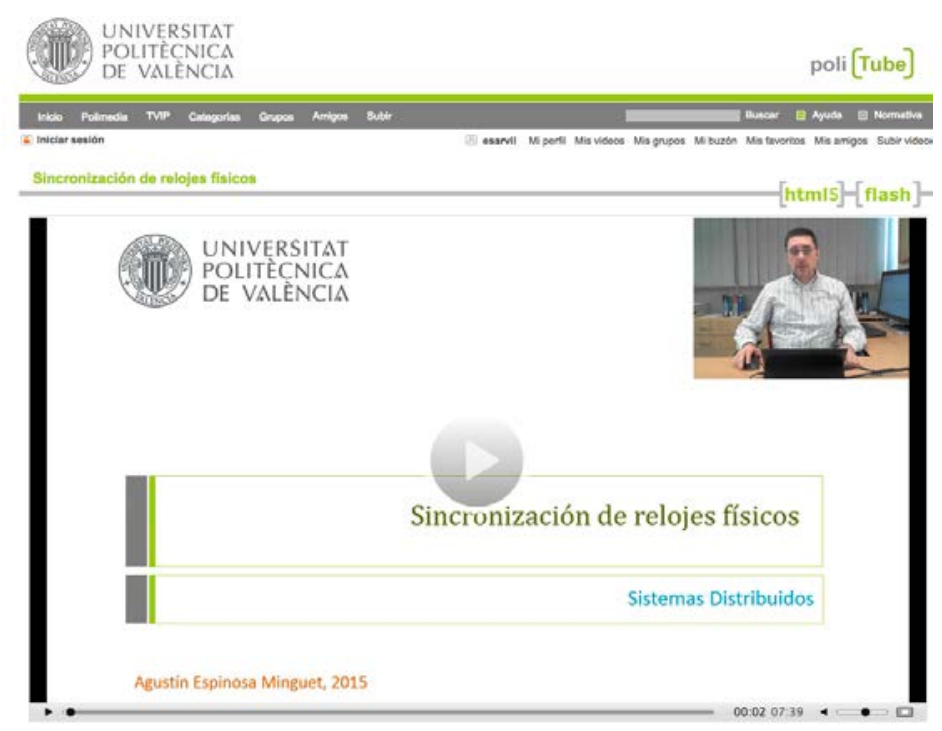

(c)) EY-NC-ND 2015, Universitat Politècnica de València 
- Elaboración de tareas previas a clase. Para garantizar la correcta marcha de las clases, los profesores hemos preparado una serie de tareas entregables a través de PoliformaT, así como diversos exámenes on-line (no evaluables), que permiten a los alumnos demostrar que han visualizado el material obligatorio, así como determinar el grado de comprensión de dicho material. Por ejemplo, por cada vídeo solicitado previamente a una sesión de aula, los alumnos debían realizar un breve resumen del vídeo y/o confeccionar un listado de unas 5-8 preguntas de tipo test (indicando la respuesta correcta), o bien debían contestar a una serie de preguntas sobre conceptos teóricos, proponer ejemplos distintos a los mostrados en el vídeo, así como indicar las dudas surgidas.

El propósito de este conjunto de tareas era, por un lado, conseguir que el alumno visualizara los vídeos y tratase de comprenderlos. Por otro lado, al ser tareas entregables (en muchos casos, con nota asociada), los alumnos recibían un feedback con las correcciones del profesor, de modo que podían clarificar sus dudas, incluso antes de asistir a las sesiones de aula correspondientes.

- Elaboración de la guía de actividades previas a clase. Antes de cada sesión, los alumnos debían visualizar un conjunto de vídeos y realizar una serie de actividades o tareas (publicitadas en PoliformaT- Tareas). Para facilitar su trabajo, por cada unidad temática se elaboró un documento explicativo con la relación de vídeos de consulta obligatoria, las tareas a realizar por cada vídeo y la fecha límite para entregar dichas tareas (generalmente, antes de la sesión de clase asociada). Además, en la guía de trabajo se incluía información complementaria, como la relación de capítulos del libro de la asignatura a consultar, enlaces a vídeos de consulta opcional, referencias de artículos, etc.

- Revisión de los boletines de problemas existentes. Se han revisado con detalle el conjunto de problemas y cuestiones disponibles de años anteriores, para así determinar cuáles podían resultar adecuados para realizar en el aula, en grupos de 2 - 4 alumnos, y añadir todas aquellas cuestiones que fueran necesarias. En concreto, por cada resultado de aprendizaje de la asignatura se han seleccionado y/o diseñado diferentes cuestiones y problemas a tratar en las sesiones de aula. De este modo, nos asegurábamos que todas las competencias y resultados de aprendizaje de la asignatura se cubrían también en el grupo de Flipped-Teaching.

En cada actividad, se ha descrito claramente su objetivo a cubrir (relacionado directamente con uno o más resultados de aprendizaje). De este modo, los alumnos también pueden comprobar que todos los resultados de aprendizaje se cubren con 
Experiencia de la aplicación de la metodología Flipped-Teaching en la asignatura Concurrencia y Sistemas Distribuidos

las actividades propuestas. Los nuevos boletines confeccionados se han puesto también a disposición del resto de grupos de la asignatura.

En las sesiones de Flipped-Teaching se ha dispuesto de tiempo suficiente para realizar todos los ejercicios propuestos (incluso muchos alumnos han considerado que el tiempo empleado en algunos ejercicios ha sido excesivo). Sin embargo, en el resto de grupos de la asignatura, se ha realizado una selección de ejercicios del boletín de problemas, ya que al invertirse bastante tiempo de aula en las sesiones magistrales tradicionales, los profesores han tenido que proponer como trabajo para casa (no entregable ni evaluable) varias cuestiones por cada unidad temática.

- Planificación de las sesiones de aula. Con antelación al comienzo del curso, los profesores realizamos un análisis de cuántas sesiones de aula eran necesarias, así como cuál debía ser la estructura general de dichas sesiones. Decidimos que, al emplear la metodología de clase inversa, no era necesario hacer uso de las 15 semanas docentes, sino que podríamos reducir la asistencia de los alumnos y con ello reservarles tiempo para la visualización de los vídeos y la realización de las tareas asociadas. De este modo, al principio de curso se proporcionó a los alumnos de Flipped-Teaching un calendario con las sesiones de aula concretas, pasándose a disponer de 11 semanas docentes (un total de 22 sesiones, en vez de 30). Con ello, las dos primeras semanas los alumnos no tuvieron clase de la asignatura y dispusieron de tiempo suficiente para visualizar los vídeos de las tres primeras unidades. Además, la clase típica de presentación de la asignatura se eliminó, pasándose también a modo "flipped” (es decir, se confeccionó un vídeo de presentación de la asignatura y del método de evaluación para el grupo FlippedTeaching).

- Publicitación del material. En el PoliformaT de la asignatura, en el apartado de "Recursos", se ha creado una carpeta llamada "Grupo Flipped-Teaching”, con todo el material y guías de trabajo de los alumnos del grupo piloto. Esto ha permitido que los alumnos de dicho grupo tuvieran totalmente centralizado todo lo que necesitaban para la asignatura: enlaces a los vídeos Politube desarrollados expresamente por los profesores, enlaces a vídeos externos de YouTube, transparencias generales de la asignatura, boletines de ejercicios, guías de trabajo, etc. Los alumnos han agradecido esta centralización, ya que en otras asignaturas tenían toda la información necesaria mucho más dispersa.

(c)) EY-NC-ND 2015, Universitat Politècnica de València

Congreso IN-RED (2015) 
Además, se decidió permitir el acceso a la carpeta "Grupo Flipped-Teaching” a todos los alumnos de la asignatura, para que así todos ellos pudieran aprovechar los recursos allí subidos (principalmente los vídeos) en su proceso de aprendizaje.

Finalmente, la asignatura CSD se evalúa a través de un conjunto de exámenes de tipo test. En concreto, los alumnos deben realizar 4 tests en el aula (con un peso del 20\% sobre la nota final) y dos exámenes parciales (con un peso del $40 \%$ sobre la nota final, cada uno).

Para el grupo piloto de Flipped-Teaching se decidió que tuviera una evaluación similar al resto de grupos, aunque también se les valorarían las actividades y tareas realizadas. De este modo, el peso de los tests en aula se redujo al $10 \%$, quedando el $10 \%$ restante para calificar, de forma conjunta, todo el trabajo realizado, tanto en las sesiones de aula como en horario no lectivo. En esta metodología resulta fundamental primar de algún modo toda la carga de trabajo que conlleva.

\section{Evaluación de la experiencia y Resultados}

En el curso 2014/15, el promedio de alumnos matriculados ha sido de 57 alumnos en los 5 grupos de mañana (uno de ellos en valenciano y otro en inglés); y de 38 alumnos en los 2 grupos de tarde. Por su parte, en el grupo de Flipped-Teaching hay 16 alumnos matriculados. En total, en la asignatura hay 379 alumnos matriculados, de los cuales 349 realizaron el primer parcial (lo cual es indicativo de que están interesados en la docencia de la asignatura).

Para evaluar esta experiencia de innovación docente se elaboró una encuesta a rellenar de forma anónima por los alumnos participantes, consistente en cuestiones para valorar la organización, planificación y la metodología de trabajo utilizados, los materiales didácticos desarrollados, el uso de las TIC, y la evaluación de los aprendizajes. También se incluyeron cuestiones para identificar los aspectos de la innovación que sería necesario modificar para su mejora.

Por otra parte, dado que en la metodología utilizada se han sustituido todas las clases magistrales de presentación de contenidos por material audiovisual para fomentar el aprendizaje autónomo, consideramos necesario comparar los resultados obtenidos por los estudiantes del grupo Flipped-Teaching con los del resto de grupos de la asignatura, en las pruebas de evaluación de la asignatura comunes, con el propósito de realizar un seguimiento del rendimiento del aprendizaje autónomo.

A continuación se analizan los resultados más relevantes de la evaluación de la experiencia.

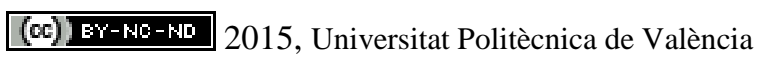

Congreso In-Red (2015) 
Experiencia de la aplicación de la metodología Flipped-Teaching en la asignatura Concurrencia y Sistemas Distribuidos

\subsection{Resultados de encuestas realizadas}

A los alumnos del grupo Flipped-Teaching les pasamos una encuesta sobre dicha metodología y su aplicación en nuestra asignatura, que fue completada por 12 alumnos (es decir, un 75\% de los matriculados). Indicamos a continuación un resumen de las respuestas recibidas, agrupadas en tres categorías:

\section{a) Aspectos positivos a destacar de la metodología "Flipped-Teaching”}

Los alumnos indican que dicha metodología les permite un seguimiento continuo de las asignaturas, llevar al día las materias, plantearse dudas antes de las clases y así poder aprovechar mejor las sesiones de aula para resolverlas, poder aprender a su propio ritmo y reducir el estrés de los exámenes. Además, los vídeos pueden verlos las veces que necesiten, en cualquier lugar y en cualquier momento, por lo que el aprendizaje se realiza cuando ellos se sienten más motivados. Esto les permite enfrentarse a las asignaturas de un modo más adecuado y consideran que así las asignaturas se hacen mucho más interesantes.

\section{b) Aspectos negativos a destacar de la metodología "Flipped-Teaching"}

Los alumnos consideran que la metodología Flipped-Teaching implica un mayor esfuerzo y una mayor sobrecarga, al requerir que los alumnos realicen determinadas tareas (visionado de vídeos, resúmenes, resolución de cuestiones, etc.) previamente a cualquier sesión de clase. Esto les crea cierta tensión al principio, ya que en la metodología tradicional el alumno no debe preparar nada, simplemente acude a las clases y se convierte en un mero receptor de las mismas. Pero con Flipped-Teaching, el alumno debe siempre llevar las clases bien preparadas. Esto les genera mucha carga de trabajo, especialmente si cursan varias asignaturas con esta metodología. Según indican, en este caso si un alumno no puede prepararse la clase, entonces asistir a la misma puede ser tiempo perdido, ya que no podrían realizar los ejercicios ni comprender las dudas que se planteen en clase, al no conocer todavía la materia tratada.

Por otro lado, los alumnos consideran que las asignaturas deberían proporcionar tablas orientativas con el tiempo necesario a dedicar en cada unidad didáctica. Además, las asignaturas implicadas deberían coordinarse para que el ritmo de trabajo no fuera tan irregular, pues consideran que existen semanas en las que tienen una carga muy elevada de trabajo, con mucho material visual y actividades a realizar en casa, mientras que otras semanas apenas tienen carga. Asimismo, la duración de los vídeos debería controlarse, siendo lo ideal vídeos de 10-15 minutos, pues mucho más tiempo lo consideran excesivo.

Finalmente, esta metodología requiere también de un alto grado de implicación por parte de los profesores, ya que deben realizar una buena planificación, tanto del trabajo autónomo como de las sesiones de aula, para así conseguir un buen ritmo de trabajo por parte de los alumnos y que el aprovechamiento de las sesiones sea máximo.

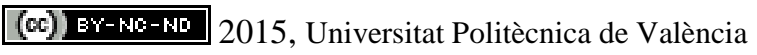


c) Valoración de los materiales didácticos desarrollados, el uso de las TIC, y la evaluación de los aprendizajes en la asignatura. Propuestas de mejora.

Los alumnos han valorado muy positivamente el material que les hemos suministrado, tanto por su variedad (consideran que había muchos medios para estudiar cada unidad, en concreto, diferentes vídeos, transparencias, un libro base de la asignatura escrito por los propios profesores), como por su calidad (consideran que los vídeos han sido concisos, claros y muy explicativos).

Asimismo, las actividades de clase les han parecido adecuadas, ya que les permitían trabajar en grupos los conceptos de la materia y les ayudaban a comprender mejor algunos aspectos. Como punto negativo, algunos alumnos consideraban que el tiempo empleado en clase para realizar los ejercicios era algo excesivo, de modo que se podría haber realizado mayor cantidad de actividades en ese mismo tiempo.

Como propuestas de mejora, los alumnos sugieren la utilización generalizada de exámenes online de preguntas tipo test para autocorrección (aspecto que se empleó en la segunda parte de la asignatura, pero que se podría ampliar al resto); la elaboración de vídeos concretos sobre ejercicios; así como una notificación constante de las tareas a realizar. En este último aspecto, conviene indicar que a los alumnos se les suministraba la guía de trabajo de cada unidad, donde se detallaba claramente los deadlines de cada tarea. Pero para algunos alumnos esto no parecía suficiente y reclamaban que se les notificase semana a semana qué debían realizar.

\subsection{Resultados de las pruebas de evaluación de la asignatura realizadas}

En el momento de realización de este artículo, el curso académico no ha finalizado todavía, por lo que disponemos solamente de resultados parciales de evaluación. En concreto, hasta la fecha se han realizado tres exámenes de tipo test en aula (distintos por cada grupo, pues el profesor responsable de grupo se ha encargado de su elaboración), así como un examen parcial, de teoría y prácticas, del primer bloque de la asignatura.

Para la comparativa de resultados del grupo de Flipped-Teaching respecto al resto de grupos (que siguen una metodología tradicional de trabajo), utilizaremos solamente los resultados del primer parcial de la asignatura, al tratarse de un examen de tipo test igual para todos los grupos.

Dicho examen constaba de dos partes: teoría y prácticas. La parte de teoría, con un peso del $75 \%$ de la nota, contenía 50 cuestiones de Verdadero/Falso y cada pregunta errónea eliminaba una pregunta acertada, aunque los primeros 5 errores no penalizaban. La parte de prácticas, con un peso del 25\% de la nota, constaba de 8 cuestiones de Verdadero/Falso y en este caso el primer error no penalizaba. 
Experiencia de la aplicación de la metodología Flipped-Teaching en la asignatura Concurrencia y Sistemas Distribuidos

En la Tabla 1 se indican los resultados obtenidos en dicho parcial, por cada grupo de la asignatura.

Como puede observarse, el primer parcial salió en general bastante mal, con un porcentaje de alumnos aprobados del 33\% sobre los matriculados, y del 36\% sobre los presentados (se presentaron al examen 348 alumnos, de un total de 379 matriculados).

Sin embargo, cabe destacar que el grupo de Flipped-Teaching obtuvo unos resultados relevantes, con un $56 \%$ de aprobados y con una nota media de grupo superior al resto de grupos (5,60 puntos) y muy por encima de la nota media global de la asignatura.

Tabla 1. Análisis de resultados del primer parcial de la asignatura

\begin{tabular}{|c|c|c|c|c|c|c|c|}
\hline Grupo & $\begin{array}{l}\mathrm{N}^{\circ} \text { alumnos } \\
\text { (matriculados) }\end{array}$ & $\begin{array}{l}\mathrm{N}^{\circ} \text { alumnos } \\
\text { (presentados } \\
\text { al parcial) }\end{array}$ & $\begin{array}{l}\text { Nota } \\
\text { Media }\end{array}$ & $\begin{array}{l}\text { Nota } \\
\text { Máxima }\end{array}$ & $\begin{array}{l}\text { Nota } \\
\text { Mínima }\end{array}$ & $\begin{array}{l}\text { \% Aprobados } \\
\text { (respecto a } \\
\text { matriculados) }\end{array}$ & $\begin{array}{l}\text { \% Aprobados } \\
\text { (respecto a } \\
\text { presentados) }\end{array}$ \\
\hline A & 56 & 50 & 3,77 & 7,60 & 0 & $25 \%$ & $28 \%$ \\
\hline B & 62 & 54 & 3,69 & 8,49 & 0 & $24 \%$ & $28 \%$ \\
\hline $\mathrm{C}$ & 66 & 64 & 4,50 & 9,55 & 0 & $44 \%$ & $45 \%$ \\
\hline $\mathrm{D}$ & 67 & 66 & 4,05 & 6,99 & 0 & $24 \%$ & $24 \%$ \\
\hline $\bar{E}$ & 36 & 28 & 4,19 & 9,09 & 0 & $44 \%$ & $57 \%$ \\
\hline $\mathrm{F}$ & 33 & 28 & 3,47 & 9,54 & 0 & $21 \%$ & $25 \%$ \\
\hline $\mathrm{G}$ & 43 & 42 & 4,70 & 8,80 & 0 & $44 \%$ & $45 \%$ \\
\hline FLIP & 16 & 16 & 5,60 & 9,10 & 2,1 & $56 \%$ & $56 \%$ \\
\hline TOTAL & 379 & 348 & 4,13 & 9,55 & 0 & $33 \%$ & $35,92 \%$ \\
\hline
\end{tabular}

Estos resultados resultan aún más llamativos si tenemos en cuenta los siguientes aspectos:

- Los alumnos matriculados en el grupo de Flipped-Teaching no eran precisamente los de mejor expediente. En dicho grupo se matricularon solamente unos pocos "valientes” que decidieron apostar por esta nueva metodología. En algunos casos, incluso se trataba de alumnos repetidores.

- Los alumnos de mejor expediente suelen matricularse en el grupo E, que se corresponde con el grupo ARA (Alto Rendimiento Académico), cuya docencia es íntegramente en inglés.

(c)) EY-NC-ND 2015, Universitat Politècnica de València 
- La asistencia a los grupos de tarde suele ser reducida, de modo que aunque oficialmente sean grupos de más de 30 personas, en la práctica hay sesiones con un número de alumnos similar al grupo de Flipped.

\section{Conclusiones}

La experiencia de la aplicación de la metodología Flipped-Teaching ha resultado muy positiva en la asignatura.

Desde el punto de vista de los alumnos pertenecientes al grupo piloto, su participación en clase ha sido muy activa, se han sentido muy motivados por la asignatura (como muestran en las encuestas realizadas) y además han obtenido mejores resultados en promedio que el resto de grupos de la asignatura. No obstante, para poder implementar de forma adecuada esta metodología, se requiere de una alta implicación por parte de los alumnos, ya que la carga de trabajo que supone es elevada. Por ejemplo, al grupo $\mathrm{F}$ de tarde se les planteó a mitad de curso pasar a metodología Flipped-Teaching y los alumnos se negaron, alegando que eso les implicaría “mucho trabajo” y que preferían la metodología tradicional.

Utilizar Flipped-Teaching no consiste únicamente en digitalizar todo el material de la asignatura, a través de vídeos. La prueba está en que en esta asignatura se ha dejado público todo el material, de modo que muchos alumnos de otros grupos lo están también usando para estudiar la asignatura. Lo principal de esta metodología es el trabajo continuado de los alumnos y profesores, así como su compromiso mutuo por llevar al día la carga de trabajo. Por tanto, mientras que los alumnos del resto de grupos suelen ver los vídeos tras asistir a las sesiones de clase (donde principalmente se realizan lecciones magistrales), los alumnos de Flipped deben comprometerse a visualizarlos antes de cada sesión y a entregar las tareas relacionadas. Por ello resulta fundamental la voluntariedad y compromiso del alumno en este tipo de metodología.

Desde el punto de vista del profesorado, la experiencia también ha resultado muy positiva y enriquecedora, pues desde el primer día de clase se ha interactuado con estudiantes que ya han trabajado previamente los conceptos y alcanzado una percepción y grado de madurez completamente diferente de la de los estudiantes a los que se les presenta la información por primera vez.

Por otra parte, aunque la elaboración del material didáctico ha supuesto un gran esfuerzo, proporciona un material de base sobre el que aplicar las propuestas de mejora en experiencias sucesivas, con objeto de alcanzar la planificación óptima en la asignatura, que equilibre la relación entre logros, esfuerzo y tiempo de dedicación. Consideramos también necesaria la coordinación con el resto de asignaturas que se imparten en el mismo semestre para evitar picos en la carga de trabajo de los alumnos, y la repetición de la experiencia en

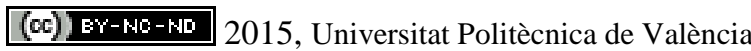

Congreso In-Red (2015) 
Experiencia de la aplicación de la metodología Flipped-Teaching en la asignatura Concurrencia y Sistemas Distribuidos

sucesivos cursos académicos y con mayor número de alumnos para poder valorarla con mayor precisión.

Por todo ello, para el próximo curso académico, además de aplicar esta metodología en el grupo especial asignado por la ETSINF que sigue esta metodología en todas las asignaturas, se está valorando su puesta en marcha en algún otro grupo proponiéndoles si desean aplicar Flipped-Teaching en esta asignatura, bien desde el principio de curso o sólo en algunas unidades.

\section{Referencias}

OLAIZOLA, A. (2014). La clase invertida: usar las TIC para "dar vuelta” a la clase. http://www.academia.edu/8350587 [Consulta: 1 de Mayo de 2015]

SÁNCHEZ RODRÍGUEZ, J., RUIZ PALMERO, J., y SÁNCHEZ VEGA, E. (2014). Las clases invertidas: beneficios y estrategias para su puesta en práctica en la educación superior. http://hdl.handle.net/10630/7821\#sthash.XpEFiBte.dpuf [Consulta: 1 de Mayo de 2015]

RUIZ PALMERO, J., SÁNCHEZ RODRÍGUEZ, J., y SÁNCHEZ RIVAS, E. (2014). "Flipped Classroom, una experiencia de enseñanza abierta y flexible". En XVII Congreso Internacional EDUTEC. Córdoba, pp. 1 - 10.

JINLEI, Z., YING, W., y BAOHUI, Z. (2012). "Introducing a New Teaching Model: Flipped Classroom” en Journal of Distance Education, vol. 4, p. 46-51. 